\title{
Controverse scientifique ou politique? Marcel Bénabou persona non grata à la Fondation Hardt
}

\section{Nicolas Gex}

\section{(2) OpenEdition \\ 1 Journals}

Édition électronique

URL : http://journals.openedition.org/edl/2439

DOI : $10.4000 /$ edl.2439

ISSN : 2296-5084

Éditeur

Université de Lausanne

\section{Édition imprimée}

Date de publication : 15 mars 2020

Pagination : 197-200

ISBN : 978-2-940331-73-4

ISSN : 0014-2026

\section{Référence électronique}

Nicolas Gex, «Controverse scientifique ou politique? Marcel Bénabou persona non grata à la Fondation Hardt », Études de lettres [En ligne], 312 | 2020, mis en ligne le 24 mars 2020, consulté le 17 septembre 2020. URL : http://journals.openedition.org/edl/2439; DOI : https://doi.org/10.4000/edl.2439 


\section{CONTROVERSE SCIENTIFIQUE OU POLITIQUE? MARCEL BÉNABOU PERSONA NON GRATA À LA FONDATION HARDT}

Chaque année ou presque depuis 1952, la Fondation Hardt pour l'étude de l'Antiquité classique, organise à Vandœuvres, où elle est installée, un colloque scientifique, les Entretiens sur l'Antiquité classique, réunissant sept à neuf savants autour d'une thématique définie à l'avance. Les exposés et les discussions qui les suivent sont publiés en volume peu après. Le Comité scientifique de l'institution, formé de dix à quinze spécialistes internationaux, supervise l'organisation des Entretiens; il veille à maintenir un équilibre entre les différentes disciplines des études classiques et garantit leur qualité scientifique. Les sujets sont choisis sur proposition d'un membre du Comité ou d'un savant extérieur, également désigné pour organiser l'édition en question. La séance annuelle de 1980 est le cadre d'un incident inédit.

En 1979, l'historien de l'Antiquité italien Emilio Gabba, appartenant au Comité depuis 1967, propose des Entretiens sur l'histoire administrative et économique de l'Empire romain. Cette initiative s'inscrit dans une série de travaux récents qui avaient renouvelé la question. Gabba connaissait bien le fonctionnement des Entretiens, ayant pris part à ceux de 1966 et organisé ceux de 1973. Le Comité agrée sa proposition pour 1982. Il charge le spécialiste italien de rédiger pour la réunion annuelle de l'automne 1980 un programme détaillé, comprenant une liste d'orateurs pressentis.

Comme prévu, le Comité procède à l'examen des Entretiens à venir lors de sa séance du $1^{\text {er }}$ novembre 1980. En l'absence de Gabba, retenu à Berkeley, lecture est faite du rapport qu'il a préparé: il propose de 
décliner le sujet en huit exposés. Plusieurs chercheurs confirmés ont déjà accepté d'y prendre part, dont Marcel Bénabou pour traiter de l'Afrique romaine. À ce nom, Pierre Grimal, professeur de latin et de culture romaine à la Sorbonne, bondit de son siège en colère: il oppose un véto irrévocable à la participation de ce chercheur aux Entretiens. En termes vifs, non consignés dans le procès-verbal, il estime que les positions de cet historien ne représentent pas l'opinion des chercheurs français, tout en dénigrant ses travaux. Son intervention est appuyée par sa collègue helléniste professeure au Collège de France, Jacqueline de Romilly, et par l'historien de l'Antiquité néerlandais Willem den Boer. Ne pouvant connaître l'avis de Gabba, le Comité repousse l'examen du projet à la séance de 1981. Ces Entretiens sont donc reportés à 1983 et une édition sur Sophocle est mise sur pied en urgence par de Romilly.

Gabba n'est informé qu’au printemps 1981 des décisions prises en son absence. Sur la base du procès-verbal de la séance et des explications du président du Comité, l'Italien juge que l'opposition de Grimal est de nature politique et non scientifique. Il dénonce la remise en cause de son choix et déplore l'embarras dans lequel cette situation l'a plongé visà-vis de Bénabou, qui avait accepté d'y participer. Refusant toute ingérence dans le choix des orateurs et regrettant la méthode adoptée à son égard, Gabba renonce, après quelques semaines de réflexion, à organiser des Entretiens et démissionne du Comité. Le projet, légèrement remanié, donne tout de même lieu à un colloque à la British School at Rome, avec l'active participation de Gabba et de Bénabou. Ironie de l'histoire, les actes de cette manifestation, publiés en 1986, seront acquis par la bibliothèque de la Fondation, sans qu'un membre du Comité ne s'en émeuve.

Malgré son caractère anecdotique et le peu de répercussions sur la Fondation, hormis la perte que représentait la démission de Gabba, cet épisode soulève divers enjeux et problèmes qu'il n'est pas facile d'appréhender. À cette fin, le recours à des sources d'information complémentaires, en particulier au témoignage d'acteurs (Bénabou et Bernard Grange, bibliothécaire de la Fondation et rédacteur du procès-verbal du Comité) permet d'expliciter certains non-dits des documents officiels, non sans risques de biais. L'attitude de Grimal était cohérente avec des prises de position antérieures. Lors de la soutenance de la thèse de Bénabou en 1972, Grimal, qui en était le directeur, avait fait part de son désaccord sur la méthode et sur les conclusions de cette étude, à la 
grande surprise du candidat et des autres membres du jury. La raison de cette attitude surprenante reste mystérieuse, même pour Bénabou, qui ne sera pas surpris d'apprendre par Gabba le véto opposé à sa présence aux Entretiens.

Lavis tranché de Grimal n'est pas remis en question par les autres membres du Comité. Ils l'ont estimé légitime en raison de son autorité et de sa connaissance du champ français. L'opinion unanime tant des autres spécialistes d'histoire ancienne (den Boer et Denis van Berchem qui lui aussi avait exprimé des réserves) que de l'autre membre française (de Romilly) du Comité a suggéré à leurs yeux un consensus autour du rejet de la personne de Bénabou. Les arguments de Grimal apparaissaient pertinents. De plus, déjuger un collègue, qui plus est connaisseur du sujet, en pleine séance aurait constitué une rupture des usages au sein de cet organe.

Gabba a mis ce refus sur le compte d'une opposition politique, sans doute en raison de sa connaissance des débats historiographiques et politiques contemporains et de leurs acteurs. La thèse de Bénabou avait en effet connu une réception mouvementée en France. Fondée sur un examen rigoureux des sources, elle rompait avec l'historiographie coloniale (souvent prétexte à justifier l'impérialisme), tout en se tenant à distance des excès des écoles nationalistes des pays anciennement colonisés. À l'aide du concept de résistance appliqué aux domaines militaire et culturel (y compris religieux), Bénabou s'intéressait aux réactions des populations africaines à la présence de Rome. Malgré un travail solide aux conclusions nuancées, salué par de nombreux comptes rendus en France et à l'étranger, cette recherche a suscité de vives discussions dans le monde scientifique français. Elle a ainsi été caricaturée, réduite à une dialectique révolte/romanisation dans l'air du temps et accusée de minimiser, voire de nier, l'apport de Rome à l'Afrique. La preuve manifeste de sa dimension militante résidait dans son titre (La résistance africaine à la romanisation), jugé provocateur, et dans le choix de l'éditeur de la version commerciale, François Maspero, connu pour son engagement anticolonialiste. D'un autre côté, les tenants d'une approche marxiste (qui n'était pas celle de Bénabou) ont également mis en cause certaines vues exprimées, avec certes moins de virulence.

Ces polémiques étaient le signe du malaise entourant la question (post)coloniale dans la société française du dernier tiers du $\mathrm{XX}^{\mathrm{e}}$ siècle. Les travaux sur l'Afrique romaine étaient particulièrement exposés et 
les savants qui s'y sont frottés ont essuyé des réactions épidermiques de certains collègues, davantage guidées par des considérations politiques que scientifiques. Cette dimension critique a-t-elle suscité la réaction de Grimal? Difficile tant à l'affirmer qu'à l'écarter; il n'apparaît pas ouvertement comme un partisan de l'Algérie française au sein de l'université parisienne, à la différence de plusieurs collègues antiquisants qui avaient signé un appel en ce sens en 1956. Il incarnait, surtout après 1968, une certaine forme de mandarinat à la tête de l'Institut des études latines, réputé conservateur. Son véto traduit peut-être des tensions internes au champ académique parisien : conflit de génération entre Grimal et Bénabou (nés en 1912 et 1939) ou entre deux institutions, la Sorbonne et Paris-VII, où ce dernier enseignait, soit entre des bastions conservateurs et progressistes. À ces facteurs s'ajoute la dimension des relations entre personnes, qui échappent à l'historien. Quoi qu'il en soit, cet incident évoque le caractère sensible des recherches sur l'Afrique romaine dans le champ académique français et la passion qui les entourait en 1980 encore.

Nicolas GeX

Section d'histoire, Faculté des lettres, Université de Lausanne

\section{BIBLIOGRAPHIE}

Courriels avec Marcel Bénabou, mai 2019.

Entretiens avec Bernard Grange, avril-mai 2019.

Archives de la Fondation Hardt, Vandouvres.

Bénabou, Marcel, La résistance africaine à la romanisation, Paris, Maspero, 1976.

Gex, Nicolas et al., La Fondation Hardt, sous la direction de Pierre Ducrey, Vandœuvres, Fondation Hardt, 2016.

Reig, Christophe, Schaffner, Alain (dir.), Marcel Bénabou, archiviste de l'infini, Paris, Presses Sorbonne Nouvelle, 2015.

Rey, Sarah, "Ne pas quitter "la terre d'Afrique". Les Antiquisants, l'Algérie française et la déclaration du 23 mai 1956 ", Anabases, 15 (2012), p. 71-84. 